Supporting Information for

\title{
Impact of Lipid Peroxidation on the Response of Cell Membranes to High-speed Equibiaxial Stretching. A Computational Study
}

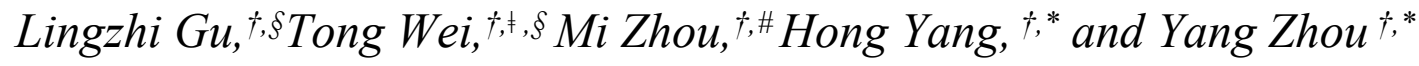

$\dagger$ Institute of Chemical Materials, China Academy of Engineering and Physics, Mianyang 621900, China.

${ }^{\ddagger}$ CAS Key Laboratory of Mechanical Behavior and Design of Materials, Department of Modern Mechanics, University of Science and Technology of China, Hefei 230026, China;

\# School of Materials Science\&Engineering, Beijing Institute of Technology, Beijing 100081, China. 


\section{Force field parameters}

The parameters required to describe the oxidized group (-OOH) were derived from quantum chemistry calculation based on $\mathrm{ffTK}^{1}$ and Gaussian $09^{2}$ programs, according to the following protocol:

(1) We first obtained the LJ parameters of the -OOH groups from the CGenFF, the parameters of oxygen and hydrogen atoms were O (OG301) and H (HGP1), respectively.

(2) Using the model molecule shown in Figure S1 to calculate partial charges and bonded parameters for oxidized lipid tails. According to the methods recommended by ffTK program, we calculated the propanoic acid-water interactions using HF/6-31G* level of theory. The partial charges were obtained by ensuring the reproduction of propanoic acid-water interactions.

(3) Bond and angles parameters were computed by fitting the potential energy surface of target molecule (propanoic acid) as implemented in ffTK, and the MP2/6-31G* level of theory was utilized.

(4) The dihedral parameters were calculated by a holistic approach in which all dihedrals were simultaneously fit to a torsional potential energy surface as employed in the ffTK modul. The MP2/6-31G* level of theory was used.

Some parameters for describing the oxidized phospholipids were directly taken from CHARMM36 force field and CGenFF, and the related parameters from above-mentioned quantum chemistry calculations are presented in Table S1-S4.

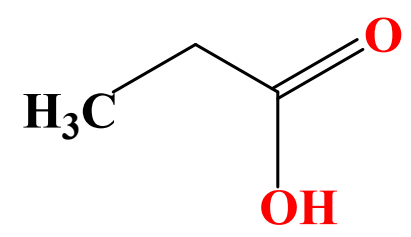

Figure S1. Illustration of the model molecule for the calculation of additional force field parameters. 
Table S1. Atomtype definition, charges and connectivity for OxPOPC.

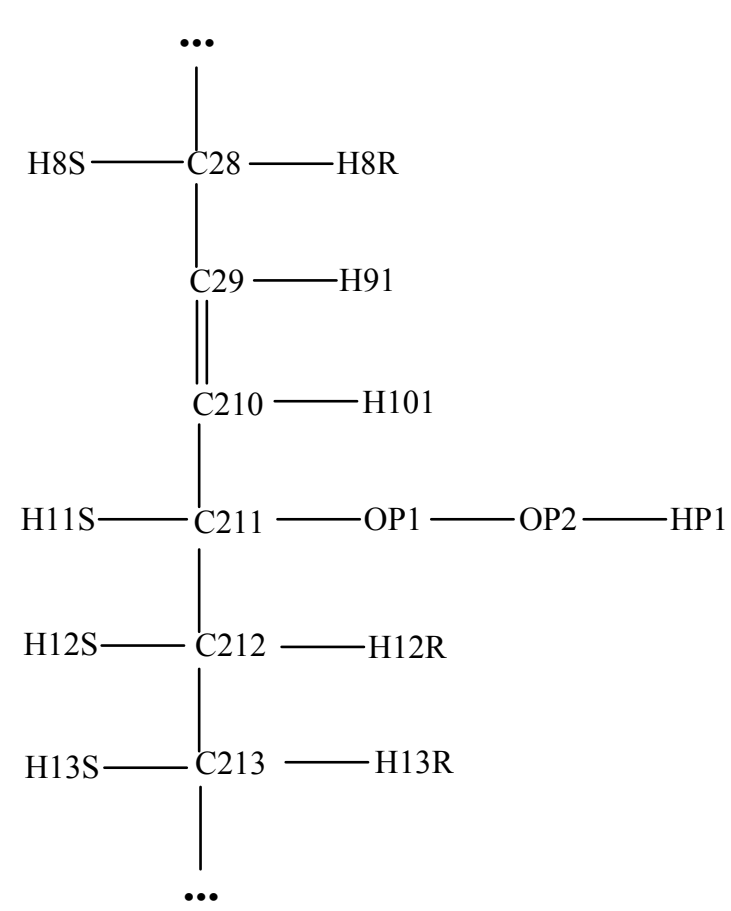

\begin{tabular}{ccc}
\hline Atom name & Atom type & charge \\
\hline C28 & CTL2 & -0.180 \\
H8R & HAL2 & 0.090 \\
H8S & HAL2 & 0.090 \\
C29 & CEL1 & -0.150 \\
H91 & HEL1 & 0.150 \\
C210 & CEL1 & -0.150 \\
H101 & HEL1 & 0.150 \\
C211 & CG311 & 0.080 \\
OP1 & OG301 & -0.138 \\
OP2 & OG301 & -0.392 \\
HP1 & HGP1 & 0.360 \\
H11S & HGA1 & 0.090 \\
C212 & CTL2 & -0.180 \\
H12R & HAL2 & 0.090 \\
H12S & HAL2 & 0.090 \\
C213 & CTL2 & -0.180 \\
H13R & HAL2 & 0.090 \\
H13S & HAL2 & 0.090 \\
\hline
\end{tabular}

Table S2. Related bond parameters used in the force field for describing the $-\mathrm{OOH}$ groups in the lipid molecules. Units are presented in the brackets.

\begin{tabular}{cccc}
\hline Atom type $\# 1$ & Atom type $\# 2$ & $\mathrm{~b}_{0}[\mathrm{~nm}]$ & $\mathrm{K}_{\mathrm{b}}\left[\mathrm{kJ} \cdot \mathrm{mol}^{-1} \cdot \mathrm{nm}^{-2}\right]$ \\
\hline OG301 & CG311 & 0.14150 & 301248.00 \\
CG311 & HGA1 & 0.11110 & 258571.20 \\
CG311 & CTL2 & 0.15380 & 186188.00 \\
CEL1 & CG311 & 0.15020 & 305432.00 \\
OG301 & OG301 & 0.14588 & 214533.00 \\
OG301 & HGP1 & 0.09726 & 364879.00 \\
\hline
\end{tabular}

Table S3. Related angle parameters used in the force field for describing the $-\mathrm{OOH}$ groups in the lipid molecules. Units are presented in the brackets.

\begin{tabular}{ccccc}
\hline Atom type \#1 & Atom type \#2 & Atom type \#3 & $\theta_{0}[$ degree $]$ & $\mathrm{K}_{\theta}\left[\mathrm{kJ} \cdot \mathrm{mol}^{-1} \cdot\right.$ degree $\left.^{-2}\right]$ \\
\hline OG301 & CG311 & HGA1 & 109.500 & 502.080000 \\
CEL1 & CG311 & CTL2 & 112.200 & 267.776000 \\
CG311 & CEL1 & HEL1 & 116.000 & 334.720000 \\
CEL1 & CG311 & HGA1 & 111.500 & 376.560000 \\
CEL1 & CEL1 & CG311 & 123.500 & 401.664000 \\
CTL2 & CG311 & HGA1 & 111.500 & 376.560000 \\
HAL2 & CTL2 & CG311 & 110.100 & 221.752000 \\
CG311 & CTL2 & CTL2 & 113.500 & 488.272800 \\
CTL2 & CG311 & OG301 & 111.500 & 376.560000 \\
CG311 & OG301 & OG301 & 106.570 & 606.109000 \\
OG301 & OG301 & HGP1 & 99.560 & 463.163000 \\
OG301 & CG311 & CEL1 & 106.730 & 536.247000 \\
\hline
\end{tabular}


Table S4. Related dihedral parameters used in the force field for describing the $-\mathrm{OOH}$ groups in the lipid molecules. Units are presented in the brackets, * refers to degree, $\neq$ refers to $\mathrm{kJ} \cdot \mathrm{mol}^{-1}$ and $\mathrm{n}$ indicates multiplicity.

\begin{tabular}{|c|c|c|c|c|c|c|}
\hline Atom type \#1 & Atom type \#2 & Atom type \#3 & Atom type \#4 & $\phi_{0}{ }^{[*]}$ & $\mathrm{K}_{\phi}^{[f]]}$ & $\mathrm{n}$ \\
\hline OG301 & CG311 & CEL1 & CEL1 & 180 & 4.439071 & 1 \\
\hline OG301 & CG311 & CEL1 & CEL1 & 180 & 0.492857 & 2 \\
\hline OG301 & CG311 & CEL1 & CEL1 & 180 & -0.429631 & 3 \\
\hline CG311 & OG301 & OG301 & HGP1 & 0 & 15.861354 & 1 \\
\hline CG311 & OG301 & OG301 & HGP1 & 0 & 7.457796 & 2 \\
\hline CG311 & OG301 & OG301 & HGP1 & 0 & 1.282340 & 3 \\
\hline CEL1 & CG311 & OG301 & OG301 & 180 & 15.471724 & 1 \\
\hline CEL1 & CG311 & OG301 & OG301 & 180 & 1.109588 & 2 \\
\hline CEL1 & CG311 & OG301 & OG301 & 180 & 1.627527 & 3 \\
\hline HGA1 & CG311 & OG301 & OG301 & 0 & 3.675480 & 1 \\
\hline HGA1 & CG311 & OG301 & OG301 & 0 & 3.084925 & 3 \\
\hline CTL2 & CG311 & OG301 & OG301 & 0 & -4.334152 & 1 \\
\hline CTL2 & CG311 & OG301 & OG301 & 0 & 0.442003 & 2 \\
\hline CTL2 & CG311 & OG301 & OG301 & 0 & 2.486251 & 3 \\
\hline OG301 & CG311 & CEL1 & HEL1 & 0 & -0.817146 & 3 \\
\hline HAL2 & CTL2 & CG311 & OG301 & 0 & -0.072525 & 3 \\
\hline HGA1 & CG311 & CEL1 & CEL1 & 180 & 3.056732 & 3 \\
\hline CTL2 & CG311 & CEL1 & CEL1 & 180 & 5.115814 & 1 \\
\hline CTL2 & CG311 & CEL1 & CEL1 & 180 & -2.010233 & 2 \\
\hline CTL2 & CG311 & CEL1 & CEL1 & 180 & 0.128741 & 3 \\
\hline HGA1 & CG311 & CEL1 & HEL1 & 0 & 2.073128 & 3 \\
\hline CTL2 & CG311 & CEL1 & HEL1 & 0 & 0.440828 & 3 \\
\hline HAL2 & CTL2 & CG311 & CEL1 & 0 & 0.523045 & 3 \\
\hline HAL2 & CTL2 & CG311 & HGA1 & 0 & 1.008433 & 3 \\
\hline CEL1 & CG311 & CTL2 & CTL2 & 0 & -6.659493 & 1 \\
\hline CEL1 & CG311 & CTL2 & CTL2 & 180 & 1.415942 & 2 \\
\hline CEL1 & CG311 & CTL2 & CTL2 & 180 & 1.738019 & 3 \\
\hline OG301 & CG311 & CTL2 & CTL2 & 0 & -3.199132 & 1 \\
\hline OG301 & CG311 & CTL2 & CTL2 & 0 & 0.471897 & 2 \\
\hline OG301 & CG311 & CTL2 & CTL2 & 0 & 1.677636 & 3 \\
\hline CTL2 & CTL2 & CG311 & HGA1 & 0 & 1.362736 & 3 \\
\hline
\end{tabular}

\section{Simulation parameters and Method}

\subsection{Mdp parameters used in the equilibrium simulation of POPC model}

$* * * * * * * * * * * * * * * * * * * * * * * * * * * * * * * * * * * * * * * * * * * * * * * * * * * * * * * * * * * * * * * * * * * * *$

title

define

$=$ POPC membrane md equilibrium

$=$ 


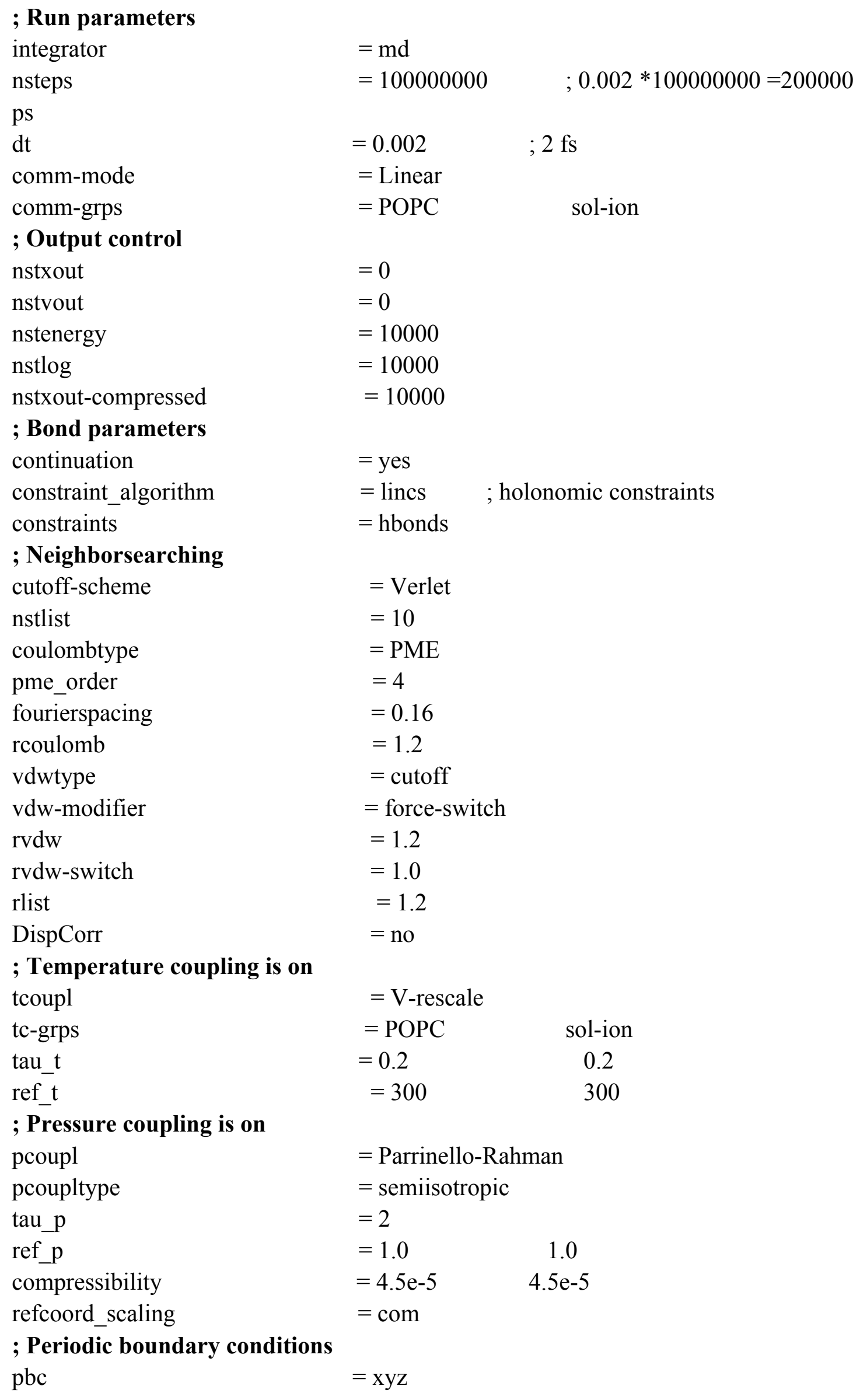




\section{; Velocity generation}

$\begin{array}{ll}\text { gen_vel } & =\text { no } \\ \text { gen_temp } & =300 \\ \text { gen_seed } & =-1\end{array}$

\subsection{The parameter sensitivity analysis in equilibrium simulation}

we modified the parameters of energy and pressure dispersion and number of LINCS iterations of the $100 \mathrm{~mol} \%$ OxPOPC model. In detail, for energy and pressure dispersion corrections, we changed it to Ener or EnerPres, and for number of LINCS iterations, we changed it to 2 , then performed equilibrium simulations on these systems, and finally calculated the structural properties of these equilibrium bilayer models, such as the area per lipid and bilayer thickness. The results are summarized in Table S5.

Table S5. Summary of parameters for the equilibrium simulations.

\begin{tabular}{lccc}
\hline \multicolumn{1}{c}{ Parameter setting } & The area per lipid $\left(\mathrm{nm}^{2}\right)$ & Bilyer thickness $(\mathrm{nm})$ \\
\hline DispCorr $=$ no; & Lincs - iter $=1$ & 0.77 & $3.24 \pm 0.04$ \\
DispCorr $=$ Ener; & Lincs - iter $=1$ & 0.79 & $3.27 \pm 0.04$ \\
DispCorr $=$ EnerPres; & Lincs - iter $=1$ & 0.77 & $3.30 \pm 0.03$ \\
DispCorr $=$ no; & Lincs - iter $=2$ & 0.78 & $3.28 \pm 0.03$ \\
\hline
\end{tabular}

\subsection{The parameter sensitivity analysis in US simulation}

we performed the sensitivity analysis about pressure coupling setting of POPC at $c=0.6 \mathrm{~m} / \mathrm{s}$, and the coupling constant tau $\_$p for Parrinello-Rahman's scaling factor was setted from 1.0 to 10.0 ps. For each scale factor, we performed five repetitions of the unsteady stretching simulations to obtain the sample average, and the results are shown in Figure S2a. Figure S2b shows all five simulation results for tau $\_p=2.0$. From Figure S2a, we can see that the increase of pore area has no obvious relationship with tau_p. Moreover, even under the same tau_p, the simulation results are different every time (Figure S2b). The reason for this phenomenon is the statistical nature of pore formation. Koshiyama et al. Pointed that a sufficient amount of water molecules in the bilayer can regulate both the probability and the route to the pore formation 
according to the number. ${ }^{3}$
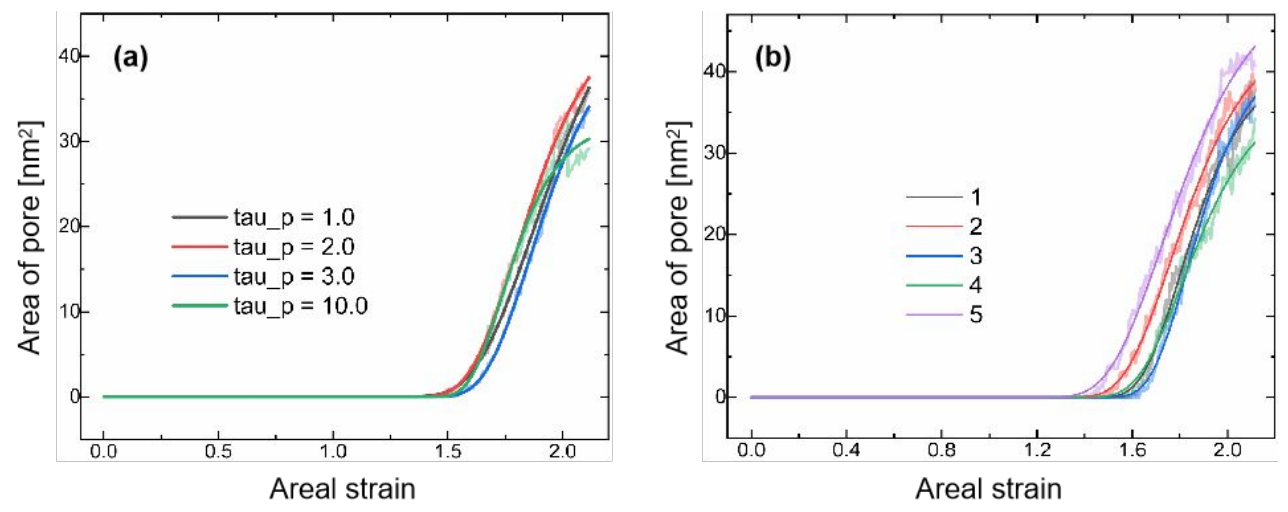

Figure S2. Pore area as a function of the areal strain for $0 \mathrm{~mol} \%$ at $c=0.6 \mathrm{~m} / \mathrm{s}$.

\subsection{Quasistatic stretching simulation.}

For comparison with the US simulations, we performed a series of constant $\mathrm{NP}_{\mathrm{z}} \mathrm{A}_{\|} \mathrm{T}$ MD (i.e., quasistatic stretching, QS) simulations of the pure POPC model. The temperature of systems and pressure in the $\mathrm{z}$ direction were maintained at $300 \mathrm{~K}$ and 1 bar, respectively. Moreover, various constant bilayer areas $A_{\|}$that was set to satisfy areal strains up to 1.0. We extracted the initial configurations from the trajectories of the US simulations with $c=0.05 \mathrm{~m} / \mathrm{s}$ and used these systems for the simulations of $100 \mathrm{~ns}$ so that the systems were equilibrated.

\section{Appendix 1. Key Parameter Calculation}

\section{Stretching modulus $\boldsymbol{K}_{A}$}

According to the following definition, the relative area $(A)$ variation consecutive to applied tension $(\sigma)$ is given by $K_{A}$ :

$$
K_{A}=\frac{1}{A}\left(\frac{d A}{d \sigma}\right)_{T, P}
$$

The elastic coefficients $K_{A}$ associated with our models come from an area fluctuation analysis (eq (2)).

$$
K_{A}=\frac{k_{B} T\langle A\rangle}{\left\langle A^{2}\right\rangle-\langle A\rangle^{2}}=\frac{k_{B} T}{4\left(\left\langle L_{x}^{2}\right\rangle-\left\langle L_{x}\right\rangle^{2}\right)}
$$

Where $\langle\mathrm{A}\rangle$ is the average value of the simple monolayer area $A=L_{x} L_{y}$, and $L_{x}$ is the average value of the lateral dimensions of the simulation box. The work of Guo et al. ${ }^{4}$ has shown that the elastic coefficient determined in this way is consistent with the 
one obtained by exerting a change in lateral stress (tension) on the membrane.

\section{Line tensions}

In our work, we assume that all of our membranes are flat and that contributions to their free energies from fluctuating undulations may be neglected. ${ }^{5,6}$ Based on these, the line tensions can be estimated as:

$$
\gamma_{\mathrm{L}}=2 K_{A}\left(\frac{\xi_{b}^{3} A_{0}}{27 \pi}\right)^{\frac{1}{2}}
$$

where $K_{A}$ is the stretching modulus and $\xi_{b}$ is the critical areal strain where a pore spontaneously closes.

To obtain the line tension $\gamma_{\mathrm{L}}$ from Eq. (3), MD simulations were divided into three stages: stretching, compressing, and equilibrating stages. The first stage was to induce the formation of pore, the second was to modulate the applied areal strain of the system with a pore, and the third was to examine whether the pore closed under the constant areal strain conditions. In the compression stage, we used the tensile structures of $0 \mathrm{~mol} \%$ and $100 \mathrm{~mol} \%$ models after $c=1 \mathrm{~m} / \mathrm{s}$, and performed MD simulations with $c=-1 \mathrm{~m} / \mathrm{s}$ until the areal strain reached 0 . And then, in the equilibrating stage, the box area was set to satisfy areal strains $\xi_{A}=0.05,0.1,0.15,0.2$, 0.25 . After the simulation with $100 \mathrm{~ns}$ and $c=0 \mathrm{~m} / \mathrm{s}$ in the equilibrating stage, we examined the existence of the pore from visual inspection and obtained the minimum areal strain where a pore remained in the bilayer and the maximum areal strain where a pore was no longer observed. The critical areal strain $\xi_{b}$, where a pore spontaneously closes, was expected to be in the range between the two areal strains. Accordingly, we estimated the upper and lower limits of the line tension via Eq (3). 


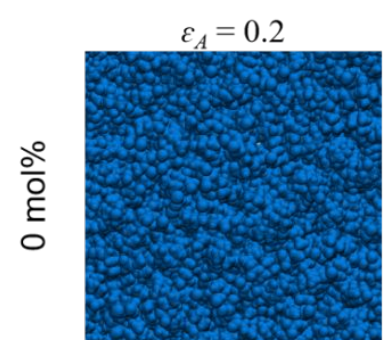

$\varepsilon_{A}=0.05$
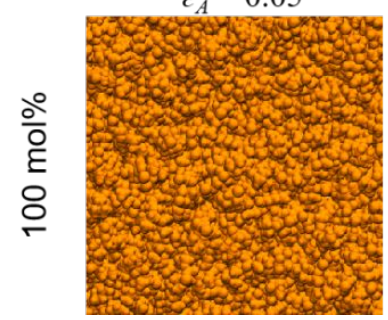

$\varepsilon_{A}=0.25$

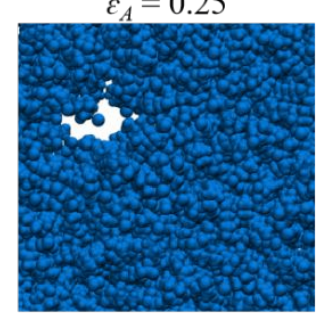

$\varepsilon_{A}=0.1$

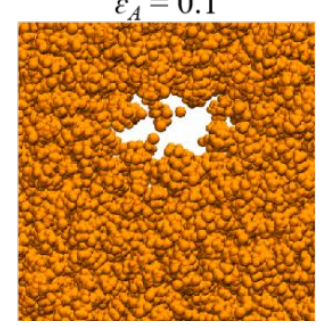

Figure S3. Representative snapshots of the bilayers including OxPOPC molecules at 0 (upper) and $100 \mathrm{~mol} \%$ (lower) after stretching, compressing, and equilibrating MD simulations.

\section{References}

(1) Mayne, C. G.; Saam, J.; Schulten, K.; Tajkhorshid, E.; Gumbart, J. C. Rapid Parameterization of Small Molecules Using the Force Field Toolkit. J. Comput. Chem. 2013, 34, 2757-2770.

(2) Frisch, M. J. E. A. Gaussian 09 Revision D.01. Gaussian Inc. Wallingford CT 2009.

(3) Koshiyama, K.; Yano, T.; Kodama, T., Self-organization of a stable pore structure in a phospholipid bilayer. Phys. Rev. Lett. 2010, 105, 018105.

(4) Guo, Y. C.; Baulin, V. A.; Thalmann, F., Peroxidised phospholipid bilayers: insight from coarse-grained molecular dynamics simulations. Soft Matter 2016, 12, 263-271.

(5) Tolpekina, T. V.; den Otter, W. D.; Briels, W. J., Simulations of stable pores in membranes: System size dependence and line tension. J. Chem. Phys. 2004, 121, 8014-8020.

(6) Shigematsu, T.; Koshiyama, K.; Wada, S., Line tension of the pore edge in phospholipid/cholesterol bilayer from stretch molecular dynamics simulation. J. Biomech. Sci. Eng. 2016, 11, NO.1. 